\title{
Cryocrystal phase transitions applied as temperature standards
}

\author{
A. Szmyrka-Grzebyk and A. Kowal \\ Institute of Low Temperature and Structure Research, ul. Okólna 2, 50-422 Wroclaw, Poland \\ E-mail: A.Kowal@int.pan.wroc.pl \\ Received January 29, 2009
}

\begin{abstract}
International temperature scales define several fixed points, which temperature values are determined with a very high precision. Some of them, which are in a low-temperature range, are called gaseous fixed points because the reference substances under a normal condition — in a room-temperature range — appear in a gaseous state. These reference substances are called by physicists cryocrystals or quantum crystals when occurring in a low temperature in a solid state. The substances play an important role in a standard thermometry. The triple point of argon, oxygen, neon and hydrogen are primary fixed points of the International Temperature Scale of 1990 (ITS-90) [1]. The triple points of nitrogen, methane and carbon dioxide are secondary fixed points of the scale [2]. Also, the solid-solid phase transitions in the cryocrystals are secondary fixed points of the scale. The highest accuracy methods of measurements applied in a standard thermometry enable the determination with a very small uncertainty of the phase transition temperatures, its reproducibility and stability. The reproducibility and stability of the transition temperature are the most important factors deciding about usefulness of these points in thermometry. Using the highest accuracy methods, better than $1 \mathrm{mK}$, which at present are used in metrology only can give a new information about nature and properties of investigated substances important also for physicists.
\end{abstract}

PACS: $64.60 . E j \quad$ Studies/theory of phase transitions of specific substances;

64.75. -g Phase equilibria;

67.90.+z Other topics in quantum fluids and solids.

Keywords: fixed point, phase transition, temperature scale, temperature standard, cryocrystals.

\section{Temperature scale}

Temperature, one of the fundamental physical quantities, cannot be measured in the same simple way as other fundamental quantities - for instance length. It is not possible to define an «unit interval» of temperature, which, multiplied, would give a numerical value for any temperature. Temperature is not an additive quantity and needs establishing a certain scale. The temperature scale must be defined by four constitutive elements:

- units,

- interpolating instruments,

- a interpolating equal,

- set of reference points.

A unit of the temperature is the kelvin, symbol $\mathrm{K}$, defined as the fraction $1 / 273.16$ of the thermodynamic temperature of the triple point of water; the degree Celsius, symbol ${ }^{\circ} \mathrm{C}$, is by definition equal in magnitude to the kelvin.
In a very wide temperature range - from $13 \mathrm{~K}$ up to about $1000 \mathrm{~K}$ - platinum resistance thermometers are used as the interpolating instruments. The thermometers must be calibrated at the number of reference points in order to define a mathematical function - an interpolating equation - describing the temperature characteristic of thermometers. The interpolating equation - a relationship between a chosen physical property (for the platinum thermometer it is electrical resistance) and the thermodynamic temperature - is not calculated from the first principles of thermodynamics but is derived from experimental data. The definition must be a simple function (or a set of functions), representing the relationship within the stated accuracy. A number of reference points, in which the platinum thermometer is calibrated, must be equal to the number of free parameters, and their position must be such as to permit the best compromise between mathematical requirements and what is given by nature. 
What is a reference point in thermometry? It is an equilibrium state of a specific substance, the realization of which does not dependent on the measurement of any other quantity, but temperature [3]. The triple point of a pure substance is the best reference point because it is a first order transition with a latent heat and with a high reproducibility of the temperature value.

In the low-temperature range, the International Temperature Scale of 1990 (ITS-90) defines six reference points:

- triple point of hydrogen with temperature $13.8033 \mathrm{~K}$,

- triple point of neon $24.5561 \mathrm{~K}$,

- triple point of oxygen $54.3584 \mathrm{~K}$,

- triple point of argon $83.8058 \mathrm{~K}$,

- triple point of mercury $234.3156 \mathrm{~K}$,

- triple point of water $273.16 \mathrm{~K}$.

The numerical values of the triple-point temperatures are determined with the accuracy of $0.1 \mathrm{mK}$ !

The scale also defines - in general with less accuracy - a set of so-called secondary fixed points:

- triple point of equilibrium deuterium $18.689 \mathrm{~K}$,

- triple point of normal deuterium $18.724 \mathrm{~K}$,

$-\alpha-\beta$ transition point of solid oxygen $23.868 \mathrm{~K}$,

$-\alpha-\beta$ transition point of solid nitrogen $35.614 \mathrm{~K}$,

$-\beta-\gamma$ transition point of solid oxygen $43.796 \mathrm{~K}$,

- triple point of nitrogen $63.151 \mathrm{~K}$,

- triple point of methane $90.197 \mathrm{~K}$,

- triple point of krypton $115.776 \mathrm{~K}$,

- triple point of carbon dioxide $216.591 \mathrm{~K}$.

Some of them have been tested at INTiBS [4-7] and results of the measurements are presented in this paper.

\section{Experiment}

\subsection{Thermometric cells and cryostat}

For the realization of the thermometric fixed points the reference substance is placed in a sealed container - cell. Thermometric cells are produced by different metrological institutes in different shapes [8-10]; see Fig. 1. INTiBS uses the cell produced by IMGC and BNM/INM.

The cells are filled with a high-purity gas $(6 \mathrm{~N})$ under rather high pressure (above $(1-1.5) \cdot 10^{7} \mathrm{~Pa}$ ) at the room temperature. In cryogenic conditions the substance appears in liquid state (about $1 \mathrm{~cm}^{3}$ ) and below its triple point - in a solid state. Detail of the cells construction is described in Ref. 3. The construction ensures reproducibility and longterm stability of the fixed point temperature.

The fixed point cells are tested in an adiabatic condition. A home-made adiabatic cryostat used for the measurements at INTiBS is presented in Fig. 2.

To achieve the adiabatic conditions in the cryostat, two gold-plated copper shields are mounted around the cell (Fig. 2,b). The temperature of the shields is controlled

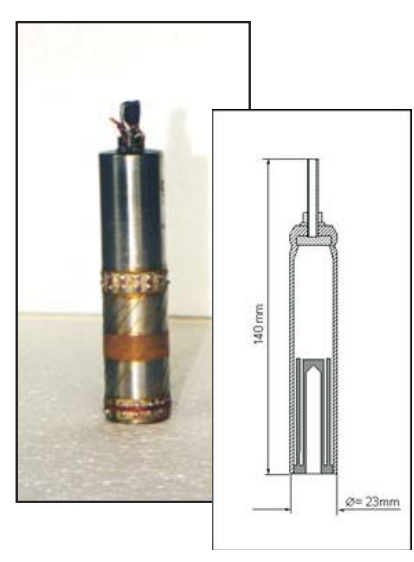

$a$

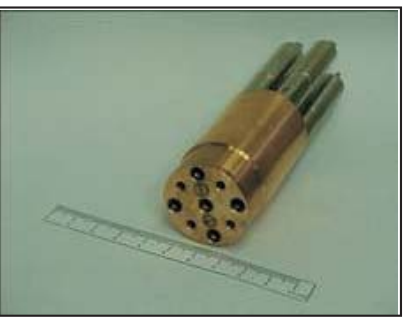

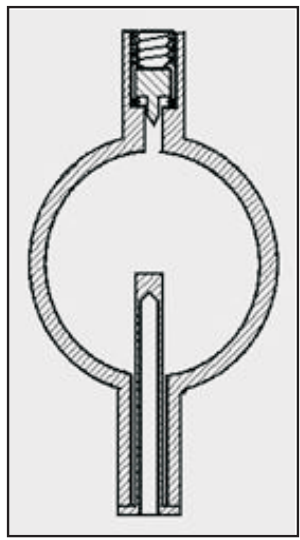

$b$

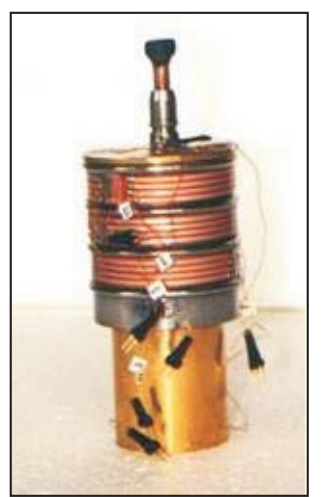

$d$
Fig. 1. Single-cell: IMGC* (Italy) (a), AIST (Japan) (b), Multicell: IMGC (Italy) (c), BNM/INM (France) $(d)$.

with $\pm 1 \mathrm{mK}$ accuracy by a good quality temperature controller (Lake Shore Cryotronics, Inc, model 340). A thermal resistance between the cell body and its surrounding, $R_{S}$, is measured by calculating the temperature drift of the cell for two different settings of the shield temperature. Using the expression:

$$
R_{s}=\frac{\Delta T_{\text {shield }}}{C\left(\left.\frac{d T}{d t}\right|_{2}-\left.\frac{d T}{d t}\right|_{1}\right)},
$$

where $C$ means the heat capacity of the cell and $(d T / d t)_{i}-$ the temperature drift of the shield in case $i$, the $R_{S}$ values were found to be in a range from $4000 \mathrm{KW}^{-1}$ for argon and $25000 \mathrm{KW}^{-1}$ for hydrogen. In these conditions the parasitic heat leak to the cell was usually less than $10 \mu \mathrm{W}$.

\subsection{Measuring methods}

The temperature of the fixed points is determined using two methods:

- continuous heating, when a heat capacity of the reference substance is estimated,

* To become: Istituto Nazionale di Ricerca Metrologica (INRIM). 


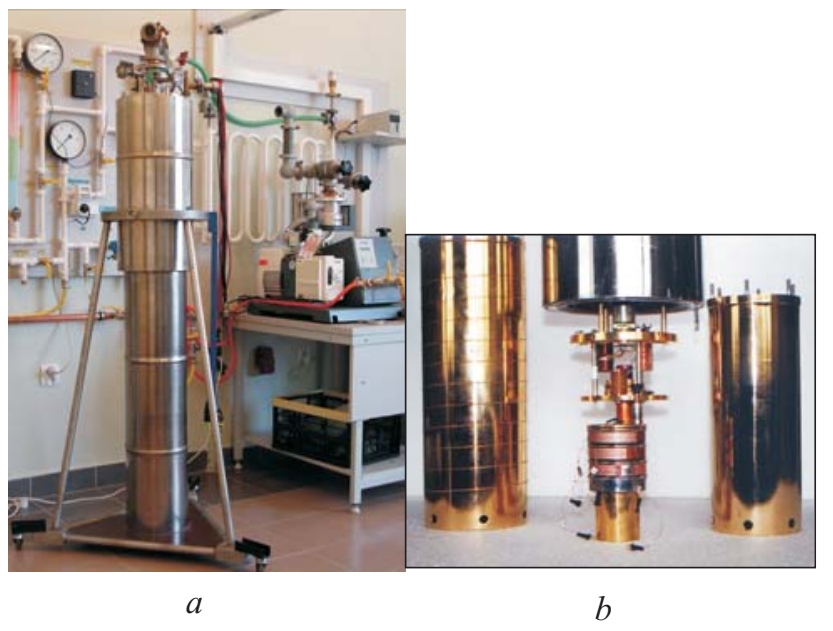

Fig. 2. Cryostat (a), measuring chamber (b).

- pulse heating, when a cell (reference substance) is heated by chosen portion of power and the triple-point temperature is defined for a state where about $50 \%$ of a reference substances is melted.

Before each determination, the cell is cooled down to the temperature of a few kelvins below the phase transition of a tested substance and kept there for several hours (10-24 h). In the first case, the cell is heated with a small constant power $(1-2 \mathrm{~mW})$ until the whole substance melts. It makes possible to calculate a total heat of melting $Q_{\text {melt }}$ for the reference cryocrystal. The value is needed for the determination of the power supplied in the pulse method of measurements. Usually, the power is chosen to melt about $10 \%$ of the substance during one heating pulse. Below the phase transition temperature, each pulse of the power increases the substance temperature. In the transition region, the temperature of the substance is almost stable because of a latent heat. When whole crystal is melted the temperature increases again after heating of the cell with the same power. After each pulse the substance temperature is measured in an equilibrium state - when the temperature is stable within about $\pm 20 \mu \mathrm{K}$.

For temperature measurements selected, very stable and reproducible $25 \Omega$ standard platinum resistance thermometers are used. The thermometers resistance is measured by high accuracy resistance bridges with the accuracy equal to $0.1 \mathrm{ppm}$. The measuring system enables temperature measurements within $\pm 0.1 \mathrm{mK}$.

\section{Results of measurements}

\subsection{Triple points}

As it was mentioned before, the ITS-90 defines six fixed points in the low-temperature range. Four of them belong to a cryocrystals family. The triple-point temperatures of these cryocrystals are determined with the accuracy of

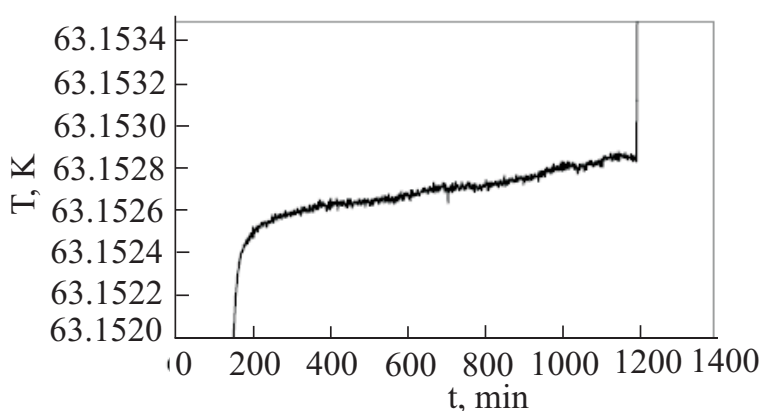

Fig. 3. The measurement of triple-point temperature of nitrogen using the continuous heating method [6].

$0.1 \mathrm{mK}$ and numerical values of the temperatures are as close as possible to the thermodynamic temperature.

Results of measurements of the triple-point temperature of nitrogen (the secondary fixed point of the ITS-90), gathered using continuous and pulse heating methods, are presented in Figs. 3 and 4. It was found that the numerical value of the triple point of nitrogen is equal to $(63.1525 \pm 0.0001) \mathrm{K}$ which is in a good agreement with the value $63.151 \mathrm{~K}$, recommended by the Consultative Committee for Thermometry (CCT) [2].

In theory, the triple-point temperature can depend on an isotopic composition of a substance. For hydrogen the relation was described in Ref. 11. The isotopic composition of natural hydrogen varies from 25 to $185 \mu \mathrm{mol} \mathrm{D} / \mathrm{mol} \mathrm{H}$. In a frame of an international cooperation several hydrogen cells with a different isotopic composition were tested $[12,13]$. Experimental results, confirming the theory, are presented in Fig. 5.

Basing on these results, recommendation of the CCT has been made to add the information to a definition of the hydrogen triple-point temperature in the ITS-90 [14].

The same effect is observed for triple point of neon. For instance, the sample-to-sample variability of the ${ }^{22} \mathrm{Ne}$ isotope amount fraction was $\sim 1.8 \%$ of the nominal value $x\left({ }^{22} \mathrm{Ne}\right)=95 \%$, corresponding to a variation in the triple point of about $0.3 \mathrm{mK}$ [15].

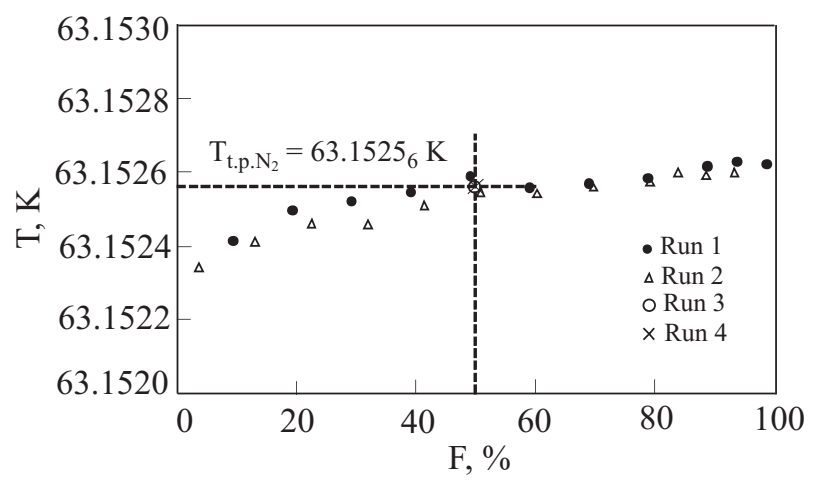

Fig. 4. Determination of the triple-point temperature of nitrogen in the pulse heating method [6]. $F$ corresponds to the fraction of melted substance. 


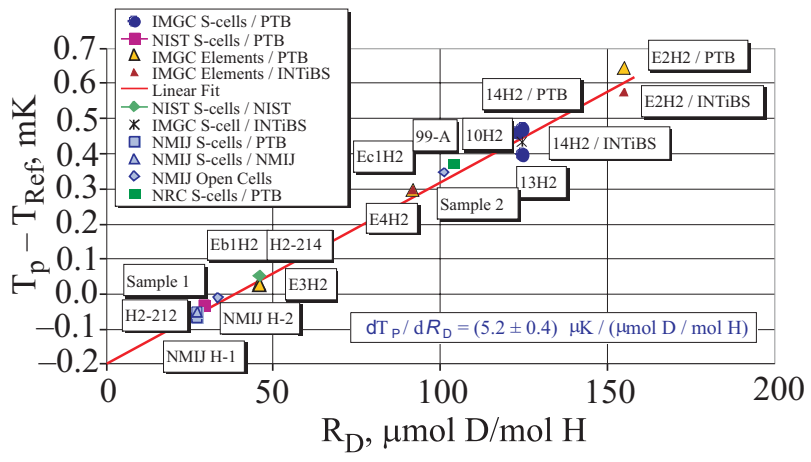

Fig. 5. The dependence of the hydrogen triple-point temperature on isotopic compositions: IMGC - Istituto di Metrologia «G. Collonetti», Italy; PTB — Physikalisch-Technische Bundesanstalt, Germany; NIST - National Institute of Standards and Technology, USA; NMIJ - National Metrology Institute of Japan; INTiBS - Instytut Niskich Temperatur i Badań Strukturalnych, Poland; NRC — National Research Council, Canada.

\subsection{Solid-solid phase transitions in cryocrystals}

The solid-solid phase transitions in two cryocrystals, nitrogen and oxygen, were tested at INTiBS. A relationship between the temperature of the substance and the fraction of a new phase formed in the cryocrystal was monitored using, when possible, both methods of measurements. Shape of the curve and reproducibility of the transition temperature were the most important factors.

\section{Oxygen}

Solid oxygen is a very unique crystal because it combines molecular and magnetic properties [16]. Two solid-solid transitions in oxygen were tested:

- the $\beta-\gamma$ transition which occurs in the temperature about $43 \mathrm{~K}$,

- the $\alpha-\beta$ transition in temperature $23 \mathrm{~K}$.

\section{The $\beta-\gamma$ transition in oxygen}

Properties of the $\beta-\gamma$ transition in oxygen were investigated using both measuring methods. The shape of the

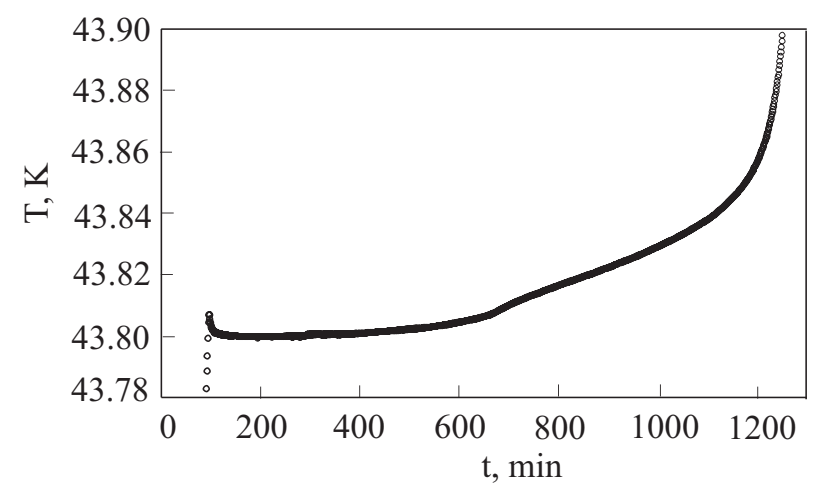

Fig. 6. The $\beta-\gamma$ transition temperature determined by the continues heating method.

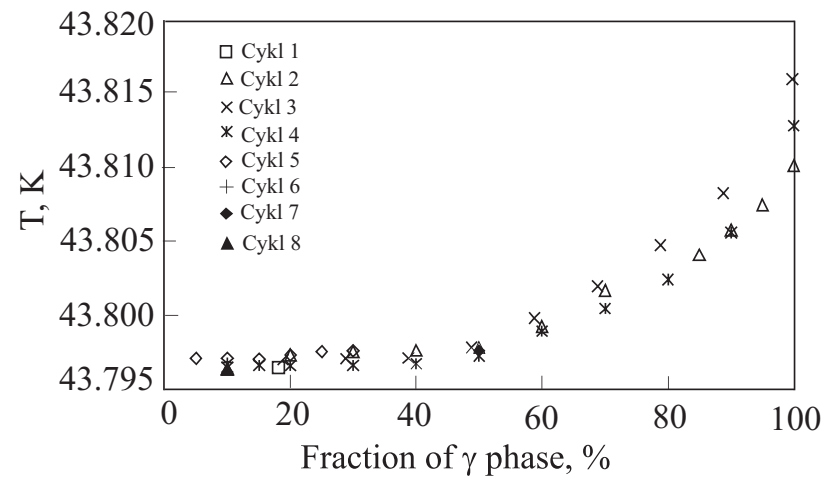

Fig. 7. Determination of the $\beta-\gamma$ transition temperature of oxygen by the pulse heating method.

curve, depicting the temperature dependence of oxygen in a fraction of the $\beta$ phase obtained during the continuous heating, is shown in Fig. 6. The same shape of the curve was observed when the heating-pulse method was used (Fig. 7).

The temperature of the crystal is stable at the beginning of the $\beta-\gamma$ transition when the most part of oxygen remains in the $\beta$ phase. When about $40 \%$ of the $\gamma$ phase is formed the temperature of oxygen starts to increase slowly and at the end of the transition is about $0.04 \mathrm{~K}$ higher than at the beginning. Beyond the transition region, when oxygen exists in the $\gamma$ phase only, the temperature of the cryocrystal increases much more quickly for the same quantity of heat supplied to the substance.

Several measuring runs performed for a flat part of the $\beta-\gamma$ transition demonstrate a good reproducibility of its temperature value at the beginning of the transition. The temperature is equal to $43.7971 \mathrm{~K}$ within $\pm 0.0006 \mathrm{~K}$ (Fig. 8).

\section{The $\alpha-\beta$ transition in oxygen}

The $\alpha-\beta$ transition in oxygen was investigated using the continuous method alone because of difficulties during the realization of the transition using the pulse method. A fundamental difficulty was a large thermal resistance between solid oxygen and the wall of the thermo-

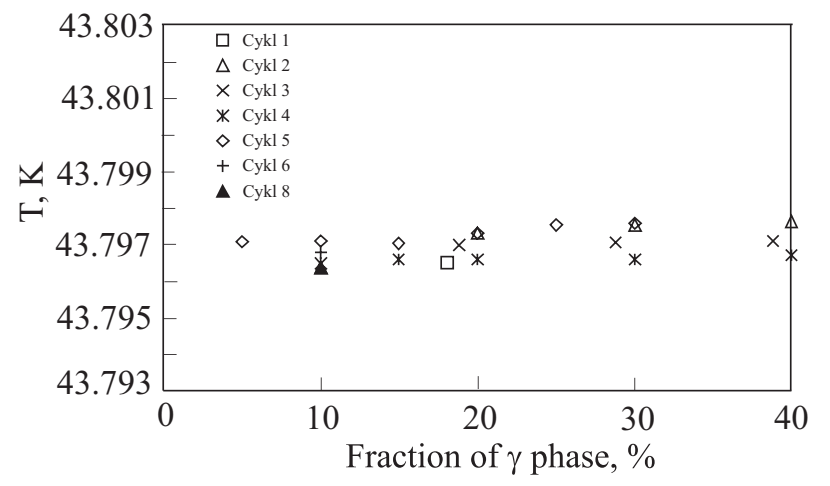

Fig. 8. Reproducibility of the $\beta-\gamma$ transition temperature for the quantity of the $\gamma$ phase less than $40 \%$. 
metric cell and the Pt thermometer. The resistance is a consequence of poor thermal conductivity of solid oxygen, particularly when it forms as a polycrystal with many defects and cracks, as it usually occurs in the thermometric cell.

The cell was always cooled down from the triple-point temperature to somewhat below the transition temperature during about $50 \mathrm{~h}$ and kept for a few hours at the lowest temperature to be sure that all oxygen transformed to the $\alpha$ phase. Then the cell was heated with the constant power equal to about $1 \mathrm{~mW}$ during about $15 \mathrm{~h}$ or longer, whereas the sample remained in the transition region no less than $5 \mathrm{~h}$, often $20 \mathrm{~h}$.

A typical shape of the curve representing the dependence of the oxygen temperature in the $\alpha-\beta$ transition region is shown in Fig. 9.

A plateau of the curve demonstrates the existence of the latent heat of the transition. An accounted value of the latent heat being about $90 \mathrm{~J} / \mathrm{mol}$ is in good accordance with the value given in Ref. 17.

From the curve the heat capacity of oxygen was calculated. The measurements were repeated several times and sharp peaks of the heat capacity are visible on the obtained curves (see Fig. 10). It needs noticing that the position of the peaks is not stable. The results show that it is difficult to determine an accurate value of the transition temperature from the curves. The total width of the transition is about $60 \mathrm{mK}$ while the temperature of the maximum of the heat capacity varies from 23.875 to $23.905 \mathrm{~K}$, without an apparent reference to other parameters of measurement [19]. The value of the $\alpha-\beta$ transition temperature in oxygen are higher that given by ITS-90 equals to $23.868 \mathrm{~K}$ with an uncertainty $5 \mathrm{mK}$.

\section{The $\alpha-\beta$ transition of nitrogen}

It is well-known that nitrogen, beyond its triple-point, exhibits also a solid-solid transition at about $35 \mathrm{~K}$. An extensive study was conducted also on the solid $\alpha-\beta$ transition of nitrogen, using both the continuous heating and the heat pulse methods at INTiBS.

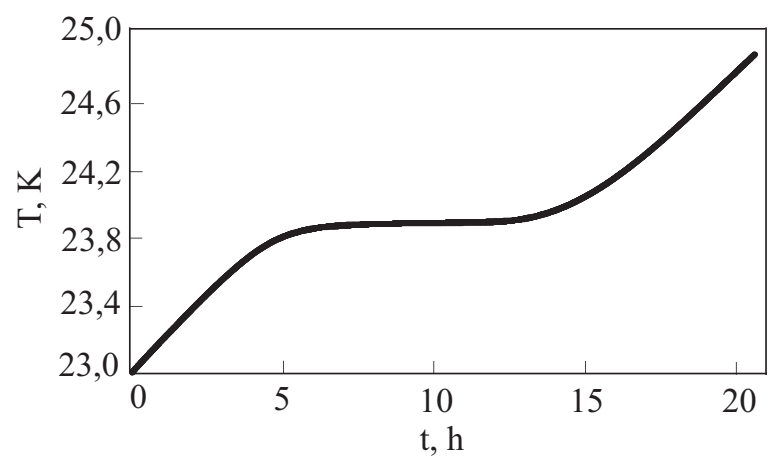

Fig. 9. The $\alpha-\beta$ transition temperature of oxygen determined in the continuous heating method.

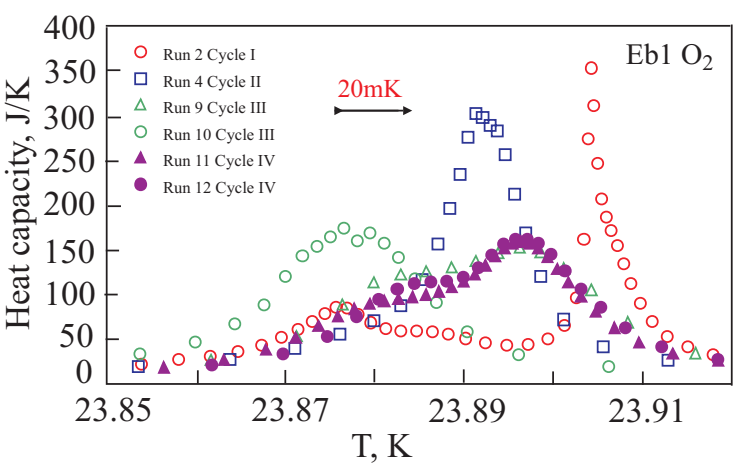

Fig. 10. Heat capacity of oxygen in region of the $\alpha-\beta$ phase transition.

The transition plateau observed during continuous heating of the sample had a very peculiar shape, not like anythat previously observed for other phase transitions (Fig. 11).

The measurements were carried out a few times with different heating rates - when the total time of the transformation varied between 5 and $15 \mathrm{~h}$. In each run the sample was cooled down below $30 \mathrm{~K}$ and kept there for a few hours. The obtained curve shapes were similar, independently of the heating rate and the «annealing» time.

The $\alpha-\beta$ nitrogen transition temperature rises to a peak before falling to a long, shallow minimum, and increase of temperature is observed towards the end of the transition. In view of this shape, is it not obvious how to associate a temperature with the transition. For thermometric purposes the central and flattest parts of the transition are the most suitable.

The temperature of the flat part of plateau of nitrogen in the $\alpha-\beta$ transition region is equal to $35.620 \mathrm{~K}$, whereas the reproducibility is better than $\pm 5 \mathrm{mK}(k=2$, were $k$ is coverage factor). This value, obtained by the continuous heating method, is in agreement with the CCT recommended value of $T_{90}=35.614 \mathrm{~K}$ with an uncertainty of $6 \mathrm{mK}(k=1)$.

Efforts were also made to determine the $\alpha-\beta$ transition in solid nitrogen using the heat pulse method. The test

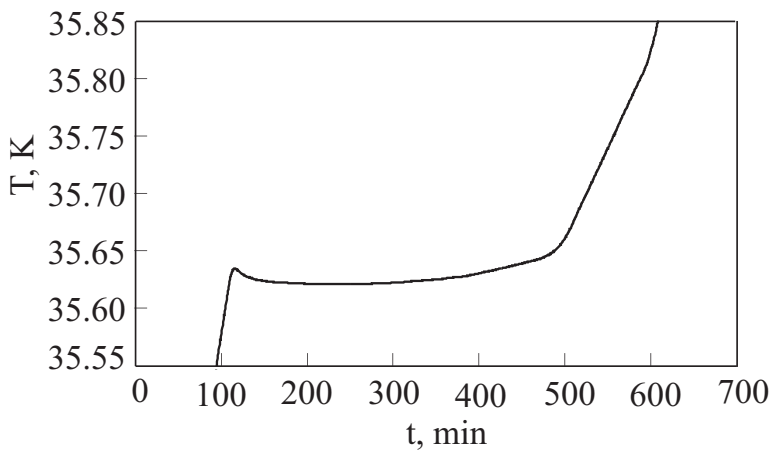

Fig. 11. The $\alpha-\beta$ transition temperature of nitrogen - the continuous heating method. 


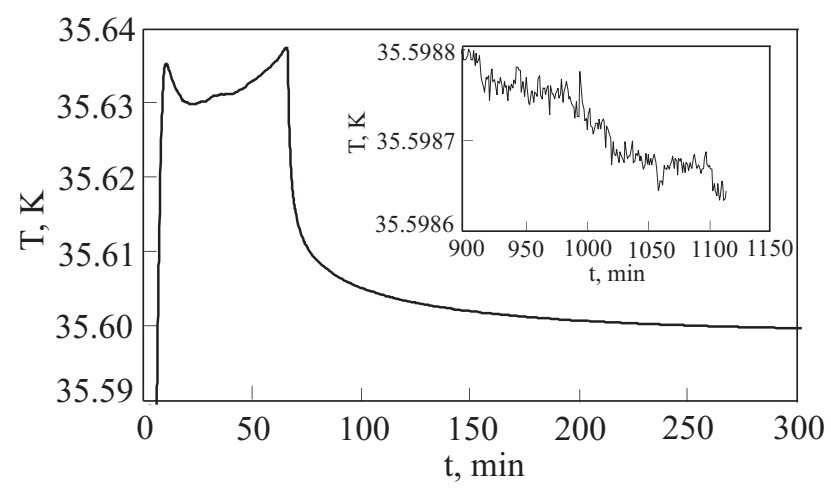

Fig. 12. Behavior of the nitrogen sample after the heat pulse in the $\alpha-\beta$ transition region.

confirmed a very large recovery time. Figure 12 shows a heat pulse and the recovery after switching off the power. As it can be seen, even after about $20 \mathrm{~h}$ the equilibrium has not been fully reached yet.

The measurements were repeated a few times, with similar results.

\section{Conclusion}

Several substances belonging to the group of cryocrystals have been tested to determine their thermometric properties. It was found that the temperatures of the triple points of argon, oxygen, neon, and hydrogen, were very reproducible and can be measured with high accuracy better than $0.1 \mathrm{mK}$. These points play an important role in thermometry defining the reference points of the International Temperature Scale of 1990.

Also the solid-solid phase transitions in some cryocrystals - like the $\alpha-\beta$ transition and the $\beta-\gamma$ transition in oxygen or the $\alpha-\beta$ transition in solid nitrogen - are proposed for the definitions of the so-called secondary fixed points of the scale. Investigation of these phase transitions has demonstrated that they are less useful as the high quality temperature standards. However the investigations, carried out with an extremely high accuracy, have exhibited these properties of the tested substances which could not be noticed in other experiments, but, nevertheless, are very interesting for physicists.

The work was partially performed with financial support from the Ministry of Sciences and High Education in Poland under project No. 505312333.
1. H. Preston-Thomas, Metrologia 27, 3 (1990).

2. R.E. Bedford, G. Bonnier, H. Maas, and F. Pavese, Metrologia 33, 133 (1996).

3. F. Pavese and G. Molinar, Modern Gas-Based Temperature and Pressure Measurements, Plenum, New York (1992).

4. L. Lipiński, A. Szmyrka-Grzebyk, and H. Manuszkiewicz, Cryogenics 36, 921 (1996).

5. A. Szmyrka-Grzebyk, L. Lipiński, and H. Manuszkiewicz, J. Low Temp. Phys. 111, 399 (1998).

6. L. Lipiński, A. Kowal, A. Szmyrka-Grzebyk, H. Manuszkiewicz, P.P.M. Steur, and F. Pavese, Metrologia 43, 435 (2006).

7. L. Lipiński, A. Kowal, A. Szmyrka-Grzebyk, H. Manuszkiewicz, P.P.M.Steur, and F. Pavese, Int. J. Thermophys. 28, 1904 (2008).

8. F. Pavese, Temperature, Its Measurements and Control in Sciences and Industry, 5, 209, American Institute of Physics, New York (1982).

9. F. Pavese, D. Ferri, I. Peroni, A. Pugliese, P.P.M. Steur, B. Fellmuth, D. Head, L. Lipiński, A. Perruzi, A. SzmyrkaGrzebyk, and L.Wolber, Temperature, Its Measurement and Control in Science and Industry, 7, 173, American Institute of Physics, Melville (2003).

10. Y. Hermier, L. Pitre, C. Geneville, A. Verge, G. Bonnier, D.I. Head, B. Fellmuth, L. Wolber, A. Szmyrka-Grzebyk, L. Lipiński, J. de Groot, and A. Perruzi, Temperature, Its Measurement and Control in Science and Industry, 7, 179, American Institute of Physics, Melville (2003).

11. N. Berezny, I. Bogoyavlenskii, L. Karnatsevich, and V. Logan, Sov. Phys.- JETP 30, 1048 (1970).

12. B. Fellmuth, L. Wolber, Y. Hermier, F. Pavese, P.P.M. Steur, I. Peroni, A. Szmyrka-Grzebyk, L. Lipiński, W.L. Tew, T. Nakano, H. Sakurai, O. Tamura, D. Head, K.H. Hill, and A.G. Steele, Metrologia 42, 171 (2005).

13. A. Szmyrka-Grzebyk, L. Lipiński, H. Manuszkiewicz, and A. Kowal, Metrology and Measurement Systems 9, 67 (2002).

14. B. Fellmuth, F. Pavese, D. Head, Y. Hermier, H. Sakurai, A.G. Steele, A. Szmyrka-Grzebyk, and W.L. Tew, Document of Consultative Committee for Thermometry CCT/01-07.

15. F. Pavese, B. Fellmuth, K.H. Hill, D. Head, Y. Hermier, L. Lipiński, T. Nakano, A. Peruzzi, H. Sakurai, A. SzmyrkaGrzebyk, A.G. Steele, P.P.M. Steur, O. Tamura, W.L. Tew, S. Valkiers, and L. Wolber, Int. J. Thermophys. 29, 57 (2008).

16. Yu.A. Freiman and H.J. Jodl, Solid Oxygen, Physics Today (2004).

17. W.F. Giauque and H.L. Johnston, J. Am. Chem. Soc. 51, 2300 (1929).

18. R.C. Kemp and W.R.G. Kemp, Metrologia 15, 87 (1979).

19. A. Szmyrka-Grzebyk, L. Lipiński, H. Manuszkiewicz, A. Kowal, and P.P.M. Steur, 2nd International Seminar and Workshop on Low-Temperature Thermometry, 119, Wrocław (2003). 